\title{
Co-sleep: Designing a workplace-based wellness program for sleep deprivation
}

\author{
Bing Zhai \\ OpenLab, Newcastle University \\ Newcastle upon Tyne \\ b.zhai2@newcastle.ac.uk \\ Yu Guan \\ OpenLab, Newcastle University \\ Newcastle upon Tyne \\ Yu.Guan@newcastle.ac.uk
}

\author{
Stuart Nicholson \\ OpenLab, Newcastle University \\ Newcastle upon Tyne \\ S.A.Nicholson@newcastle.ac.uk \\ Patrick Olivier \\ Monash University \\ Melbourne \\ Patrick.Olivier@monash.edu
}

\author{
Kyle Montague \\ OpenLab, Newcastle University \\ Newcastle upon Tyne \\ Kyle.Montague@newcastle.ac.uk \\ Jason Ellis \\ Northumbria University \\ Newcastle upon Tyne \\ jason.ellis@northumbria.ac.uk
}

\begin{abstract}
Sleep deprivation is a growing public health issue which has a range of implications on a person's working routine. Yet despite the effects of sleep deprivation, workplace-based wellness programs do not have any awareness of sleep deprivation. Our study adopted a three-stage design process with nine participants from a local manufacturing company to highlight awareness of sleep deprivation. Our study contributes design considerations for the implementation of workplace-based wellness programs that raise awareness of the importance of sleep quality as how employees can share their personal sleep data within the workplace.
\end{abstract}

\section{CCS CONCEPTS}

- Human-centered computing $\rightarrow$ Human computer interaction(HCI); • HCI design and evaluation methods;

\section{KEYWORDS}

Sleep deprivation, wearable sensors, digital civics, digital health, workplace wellness program

\section{ACM Reference Format:}

Bing Zhai, Stuart Nicholson, Kyle Montague, Yu Guan, Patrick Olivier, and Jason Ellis. 2018. Co-sleep: Designing a workplace-based wellness program for sleep deprivation. In Woodstock '18: ACM Symposium on Neural Gaze Detection, May 20-23, 2019, Trento, Italy. ACM, New York, NY, USA, 4 pages.

\section{INTRODUCTION}

Within the UK approximately one-third of the population suffer from sleep deprivation to varying degrees. Lack of sleep significantly impacts on people's work and overall quality of life. This sleep-loss epidemic has been estimated to cost the UK $£ 40$ billion annually, this is the equivalent to $1.9 \%$ of the GDP [10].

Permission to make digital or hard copies of all or part of this work for personal or classroom use is granted without fee provided that copies are not made or distributed for profit or commercial advantage and that copies bear this notice and the full citation on the first page. Copyrights for components of this work owned by others than the author(s) must be honored. Abstracting with credit is permitted. To copy otherwise, or republish, to post on servers or to redistribute to lists, requires prior specific permission and/or a fee. Request permissions from permissions@acm.org.

PervasiveHealth '19, May 20-23, 2019, Trento, Italy

(c) 2018 Copyright held by the owner/author(s). Publication rights licensed to ACM ACM ISBN 978-1-4503-6363-1/xx/xx...\$15.00
For office workers this would significantly reduce their efficiency, potentially leading to an increase in work-related stress due to the demands of having to 'catch-up' on daily workloads [7]. In general, the risks of sleep deprivation have not been widely disseminated within the UK and public awareness of sleep deprivation remains low.

Personal informatics tools and mobile applications which support sleep tracking are becoming more ubiquitous [4]. Mobile apps have been designed to assist people in improving their sleep hygiene behaviour constructed around a stage-based model [8]. When designing to track and share personal health data within workplace, these introspective models face several challenges, including pervasive data capture, and issues pertaining to personal privacy and health data sharing. Our study contributes design considerations towards the implementation of workplace-based wellness programs inclusive of employees sleep data; insight into strategies surrounding data privacy and anonymity as well as ways of limiting the potential for employer misuse of workers' sleep data.

\section{RELATED WORK}

Sleep deprivation affects the manufacturing industry in the form of diminished productivity, increased product defect rates and increased accident rates. Workers on shifts as part of a 24-hour cycle within manufacturing companies are highly prone to sleep deprivation; this can lead to circadian misalignment which is the primary factor that occurs when the body clock is altered to the extent that someone finds it difficult to fall asleep during night time [11]. Insufficient sleep can impair a person's attention to detail, alertness and concentration. Many companies implement fatigue management plans to prevent accidents. One such example includes training for workers in how to more efficiently make use of daytime sleep opportunities to adapt to a rotational shift pattern.

Choe et al.[5] highlights that people have a strong willingness to track their sleep and factors affecting or relating to their sleep in a low-burden manner; the study highlights that only by looking at longitudinal trends can people truly identify problems. A number of studies and mobile applications have explored ways of capturing sleep patterns and providing feedback and/or suggestions for sleep hygiene-related behaviours (e.g. avoid use electronics before going to bed) that relate to personal sleep health [4]. Furthermore, Pina et. al [13] extended a personal informatics system in designing for a family to track and share sleep data among its members - a 
first step towards collectively addressing sleep issues that frames interventions as social processes whereby data is shared with family members to understand the causes of lack of sleep.

Employee health promotion is an activity that employers are increasingly engaging in [6]. For example, companies subsidize their employees purchases of fitness trackers and/or gym membership. Activity trackers are personal informatics device most commonly used to collectively encourage behaviour change within the work environment as part of employer health and wellbeing campaigns [6]. Such programs can be divided into two categories, the shortterm project normally last few months and long-term projects can last more than a year. Both of them include incentives such as virtual points that can be redeemd as cash reward or gift card to encourage employee participation in events [9].

\section{PROJECT DESIGN AND METHODOLOGY}

Our aim was to explore the design and implementation of deploying a sleep wellness program within the workplace. Utilising an action research approach $[\mathrm{AR}]$ and working closely with stakeholders from a local manufacturing firm, we worked with 9 employees. The project ran across one month with three distinct stages: 1) design and deployment of digital and cultural probes for gathering accounts and understanding sleep deprivation issues; 2) running design workshops for understanding stakeholder considerations of sharing sleep related information within a workplace environment; 3) design of sleep data visualisations and conduct data-driven interviews which investigated corporate, business, and social responsibility as well as workplace and personal ethical concerns. Ethical approval was secured by the university ethical board and our stakeholder ethics board. Participant stakeholders consisted of: 2 members of senior management, 3 shift-based floor workers and 4 clerical staff which represented a cross section of roles and responsibility within the company.

Participants were invited to wear the Axivity ${ }^{\mathrm{TM}} 3$ wristbands for one week whilst recording other sleep related notes into a diary; data from the wristbands were online web form at the end of each day. Actigraphy equipment was used for the processing of our results which is widely used in ambulatory sleep monitoring studies by clinicians [1]. Our sleep data was processed by an "Estimation of Stationary Sleep-segments"[2] algorithm to generate the actigraphy data which is typically used for determining sleep pattern and circadian rhythms with an accuracy of $86 \%$. Each stakeholder was presented with an overview of their actigraphy data in the form of a visualisation within a one-to-one interview, and then asked a series of open questions by the first author to gain insight into their data and apply context. Following initial interviews, two workshops with 5 of the 9 participants were run for the purposes of understanding: 1) what type of data stakeholders prefer to collect (other than Actigraphy); 2) how they would like to collect this data and 3) how they would prefer to share it within the workplace. We adapted an existing 'Tiles IoT toolkit' [12] for our next workshop (adding custom data cards) that had been designed to present data in a variety of detail (granularity). In our third, and final stage, we designed two interactive dashboards: one dashboard displayed personal sleep data for a single stakeholder which was intended for private viewing. The second dashboard displayed all of our participating stakeholders sleep data anonymously, alongside their notes from their respective sleep diary. The interactive dashboards were designed using Qlik ${ }^{\mathrm{TM}}$ Sense, which also produces a report based on the data (Figure 1),. Interview data was analysed using a thematic approach[3]); quotes were coded inductively and summarised with short codes which were then grouped into larger candidate themes. We draw our findings from the subsequent themes that emerged from the interviews, the Actigraphy data, sleep diaries and workshop observational notes.

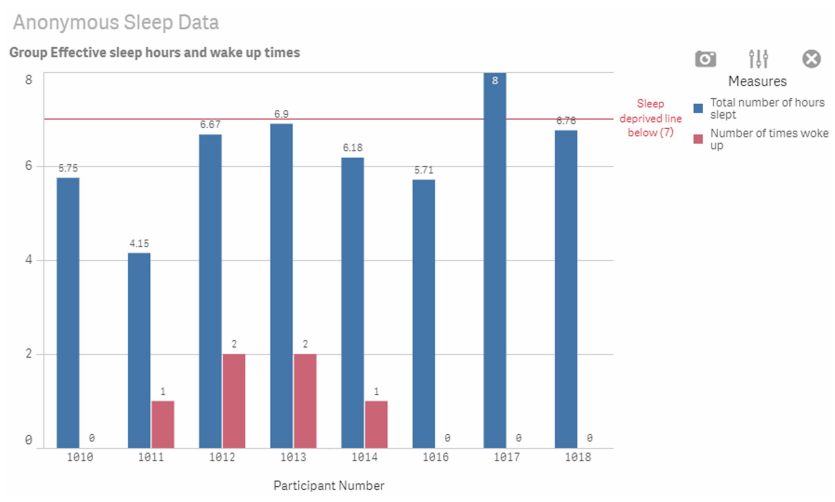

Figure 1: An example of One night sleep data dashboard, more details to see (https:/github.com/famousgrouse/ pervasivehealth).

\section{INTERVIEW FINDINGS}

During the initial consultation as part of our study, six of our nine participants were not aware they were sleep deprived; we found this by compared each participants sleep data to that of the UK Governments health department, 7-9 hours per night guideline. We observed a general lack of awareness about sleep deprivation, sleep quality and what effective sleep is; there was no notion of how much sleep they should try to get, nor that the hours of restlessness do not count as sleep. When asked, our participants highlighted the main reasons their sleep was disrupted or irregular was because of other family members or personal reasons, which resulted in their sleep deprivation. For example, they may have to provide care for other family members during the night. Work related issues also affected participants sleep; three highlighted the additional demands and pressure of work meant they were often thinking of the next days workload while lying in bed. These three participants stated that they often work overtime and regularly check work emails before going to bed. Some of our participants work shift cycles; this in itself can also lead to a lack of sleep due to the irregular routine and subsequent circadian rhythm.

As part of our interviews, we gained some insight into the perceived potential impact of implementing a wellness program. The employer (as an organisation) was perceived more favourably, even for allowing this study to take place as the initial step towards developing a workplace based wellness program. Participants were generally appreciative of their company being interested in their welfare: "I would say it's good that they are doing this"(P2). Four 
participants expressed that they had underlying concerns regarding the sharing of individuals sleep data. For example, P2 stated: "Sharing sleep data may cause others to question the life of people who sleep less, [...] or guess whether the employee's personal life will be troublesome [or if they] are mentally ill."

Within stage two of the study, we ran two identical IoT and data sharing workshops (to accommodate participants work schedules). In the first-half, we evaluated the variety of sensors that can be used for the purposes of wellness tracking. Participants were particularly uncomfortable with camera-based tracking in the workplace: "it's too invasive, too in your face type of thing and the microphone might be a little bit too invasive of your [...] personal conversations" ( $\mathrm{P} 2)$. In the second-half, we attempted to investigate how personal data should be shared within the workplace. In summary, participants preferred only sharing their daily sleep data anonymously; eight participants confirmed they were happy to share their aggregated data over an extended period of time, linked to their identity.

In our final stage, each participant was asked to review their private and public dashboard, and then answer a set of questions designed to explore their understanding and any insights they had about the data being presented. Participants were interested in their own data, but more interestingly, that of other participants; they expressed concern over their colleagues who showed less sleep. By sharing personal sleep data amongst all participants allowed them to reflect on the groups data collectively, as P8 noted: "wow, I know people don't get enough sleep, but that is pretty bad." Our interactive dashboard demonstrated how useful it could be as a tool for allowing participants to discover patterns over time: "large scale you can find a more accurate pattern" (P7). We also noted that sharing data may stimulate communication and a sense of care between colleagues within the workplace, as P3 notes: "I think it's quite good if I made that public... I am promoting people then to try and talk about my issue of lack of sleep". In running our study to develop a wellness program, the employer benefits by having an increased awareness of its employees alertness and general fatigue. As previously iterated, it also reinforced employee's perception that the company cares more about them and their welfare. P8 believed that "things like this can only improve to me what companies consider to being their responsibility".

Concerns arose from our participants over ownership and availability of sleep data. It was unclear, due to sleep taking place outside of working hours, whether the employees or employer owned the data, where it would be held, and how long it could be held for. Upon presenting participants with the dashboard, two changed their opinion about sharing sleep data publicly within the workplace; they highlighted that people should "Only share if you have a good eight hours sleep ...”(P9) alluding to the fact that your employer may treat you differently if you appear to be tired or fatigued. Participants also noted that they would prefer not to share their weekend sleep data, apart from perhaps Sunday night before work begins again. Participants recommended that the control of sensitive data should be controlled by someone who is impartial and non-bias, such as human resources (HR).

Similar to ownership, participants raised concerns over the potential miss-use of their sleep data within the workplace; how would it be used over time as well as how long could it be held for. Participants sleeping habits had a greater impact on work than any other health-related activities. When participants were asked if the company would use their data to treat their employees differently, P6 advocated that ideally, sleep data should be used productively: "seriously [you] have to look at your policies, but the first one will be education and the second one on there will be support. You wouldn't look to discipline your staff if somebody has a genuine issue". P6 instead suggests that if an employee suffered acute sleep deprivation for one night (less than two or three hours sleep), the company could acknowledge it as part of the employees sickness policy. Building on this, P7 highlighted that monitoring sleep data can also be used as a means for protecting employees and highlighting problems: "go against the company... when there's no way really that they are going to be getting the average seven hours sleep" (problems associated with general lack of sleep in shift patterns). Participants also highlighted that more substantial sleep deprivation may result in safety concerns can occur as an effect of alcohol and drug intake. P6 said that workers "had to be a certain alertness to operate the machine ... it's health and safety ...it should be done". Senior management participants believed that sleep not only impacted upon a person's physical and mental health, but also directly related to workplace-based accidents.

\section{DISCUSSION}

Drawing from our findings, we highlight three distinct areas that require addressing when designing for the implementation of workplacebased sleep wellness programs. Firstly, we address the lack of awareness surrounding sleep deprivation, sensing technology and personal data, as well as it's importance for employee wellness; Secondly, we highlight the importance of co-designing sleep wellness programs within organisations to bolster employee-employer relationships and thirdly; planning for the sustainability of a workplacebased sleep wellness program.

Throughout the initial stages of our study, we were surprised by our participants lack of knowledge surrounding the amount of sleep needed between working days. Our participants had little to no awareness of the recommended amount of sleep required, nor were they particularly aware of what constituted sleep deprivation and how it could affect them within the workplace. Some participants were aware of the implications that reduced sleep could have on their work performance, but this was typically anecdotal. Similarly, there was a general vagueness from participants surrounding the distinction of personal data which was further compounded by participants unfamiliarity with sensing technology and what different sensors or mechanisms could track. Our first recommendation is simple, but arguably very important, as it underpins the necessary awareness and understanding for sleep and its implications within the workplace. We suggest the use of a 4-stage training package as way of inducting employees across the topics of sleep, sleep deprivation, sensing technology and personal data; the four areas which our participants had limited understanding. Firstly, the training package should reiterating health guidelines and recommendations surrounding sleep and rest. Secondly, an introducing to sleep deprivation with a specific focus on its impact within the workplace should take place. This should provide insight into previously conducted workplace-based wellness tracking projects, which can then lead into thirdly; the existence and use of different sensing 
technologies for personal tracking. Fourthly, making it clear what constitutes personal data as well as defining the distinction between workplace and personal life.

As with communities, the workforce of an organisation can be unique in itself. It is apparent that what may work for one group of people, may not work at all for another. Participants responded positively to their employer allowing the study to take place, stating that it made them feel as if their employer was interested in their health and wellness. Despite this, employees did present concerns regarding the use of their personal data and how this could potentially impact on their employment. We recommend co-designing sleep wellness programs alongside employees to take account of their opinions, thoughts and concerns within a democratic process. This process not only serves the purpose of designing a bespoke program, tailored for employee and employer needs, but one which is also configured for a particular organisation or types of industry (e.g. farming, manufacturing, education \& IT); it also serves to increase visibility and understanding of the initiative amongst employees more generally which can bolster the employee-employer relationship. To mitigate issues of distrust amongst workers, we recommend the establishment of consent-based practice with regards to data access and interpretation; the use of smart contracts and token-based access for data access would serve as a transparent and secure way for both employees and employers to share data whilst maintaining mutual trust.

Echoed throughout our findings were issues relating to the longer-term sustainability of managing a workplace-based sleep wellness program which included concerns over administration, accountability and responsibility. Employees and employers alike expressed concern over who would be accountable should an accident occur as a result of someone being sleep deprived, as well as who should administer and maintain the program once it has been implemented within an organisation. Workers were keen to outline $\mathrm{HR}$ as an administrative, impartial body for the up-keep of the program however, the responsibility of employees ensuring they are getting enough sleep should arguably be mutual. Evidently the establishment of policies and guidelines relating to the long-term management, use of data and accountability need to be outlined as part of the deployment of a sleep wellness program. Moreover, there needs to be further thought towards how such programs can firstly; support employees who have demonstrated genuine sleepdeprivation issues and secondly; how sleep wellness programs can sustain employee engagement, openness and willingness to share within the workplace in order to maintain momentum. In developing organisational policies which incorporate the sleep wellness program at their core, this would establish a contractual obligation between employees and employers to engage in the program. Alternative participation based models could be designed to sustain engagement through the inclusion of gamification around sleep hygiene and quality, to encourage employee participation over sustained periods of time.

\section{CONCLUSION \& FUTURE WORK}

This study presents a series of design considerations that should be integrated as part of the process for designing a sleep wellness program which affords co-designing alongside employees. We highlight employees concerns surrounding the use of their personal data, how it is shared as well as their fears of how it could potentially be misused by employers; we also propose considerations for the sustainability of managing and maintaining a sleep wellness program.

Future work seeks to clarify issues of data ownership within the workplace environment, as well as outline the distribution of governance employers have over their employee's personal data. Further case studies should apply more formalised and established policies surrounding workplace-based wellness programs so that longer term deployments can highlight additional factors contributing to the programs sustainability, employee retention, effectiveness, usability and acceptability. These further studies will help to mould future platforms that can empower employees to take ownership of their own data and control how it is shared amongst others within the workplace.

\section{ACKNOWLEDGMENTS}

This research was funded through the EPSRC Centre for Doctoral Training in Digital Civics (EP/L016176/1). Data supporting this publication is openly available under an 'Open Data Commons Open Database License'. Please contact Newcastle Research Data Service at rdm@ncl.ac.uk for access instructions.

\section{REFERENCES}

[1] Axivity. 2018. Axivity. https://axivity.com/product/ax3

[2] Marko Borazio, Eugen Berlin, Nagihan KÃijc Ãijkyildiz, Philipp Scholl, and Kristof Van Laerhoven. [n. d.]. Towards benchmarked sleep detection with wrist-worn sensing units. In Healthcare Informatics (ICHI), 2014 IEEE International Conference on. IEEE, 125-134.

[3] Virginia Braun and Victoria Clarke. 2006. Using thematic analysis in psychology. Qualitative research in psychology 3, 2 (2006), 77-101.

[4] Eun Kyoung Choe, Bongshin Lee, Matthew Kay, Wanda Pratt, and Julie A Kientz. [n. d.]. SleepTight: low-burden, self-monitoring technology for capturing and reflecting on sleep behaviors. In Proceedings of the 2015 ACM International foint Conference on Pervasive and Ubiquitous Computing. ACM, 121-132.

[5] Eun Kyoung Choe, Nicole B Lee, Bongshin Lee, Wanda Pratt, and Julie A Kientz. [n. d.]. Understanding quantified-selfers' practices in collecting and exploring personal data. In Proceedings of the 32nd annual ACM conference on Human factors in computing systems. ACM, 1143-1152.

[6] Chia-Fang Chung, Nanna Gorm, Irina A Shklovski, and Sean Munson. [n. d.]. Finding the right fit: understanding health tracking in workplace wellness programs. In Proceedings of the 2017 CHI Conference on Human Factors in Computing Systems. ACM, 4875-4886.

[7] Anna Dahlgren, GÃüran Kecklund, and TorbjÃürn ÃĚkerstedt. 2005. Different levels of work-related stress and the effects on sleep, fatigue and cortisol. Scandinavian journal of work, environment health (2005), 277-285.

[8] Thomas Fritz, Elaine M Huang, Gail C Murphy, and Thomas Zimmermann. [n. d.]. Persuasive technology in the real world: a study of long-term use of activity sensing devices for fitness. In Proceedings of the SIGCHI Conference on Human Factors in Computing Systems. ACM, 487-496.

[9] Nanna Gorm and Irina Shklovski. [n. d.]. Sharing steps in the workplace: Changing privacy concerns over time. In Proceedings of the 2016 CHI Conference on Human Factors in Computing Systems. ACM, 4315-4319.

[10] Marco Hafner. 2016. Lack of Sleep Costing UK Economy Up to Âč40 Billion a Year. https://www.rand.org/news/press/2016/11/30/index1.html

[11] Nicola Magnavita and Sergio Garbarino. 2017. Sleep, health and wellness at work: A scoping review. International journal of environmental research and public health 14, 11 (2017), 1347

[12] Simone Mora, Francesco Gianni, and Monica Divitini. [n. d.]. Tiles: a card-based ideation toolkit for the internet of things. In Proceedings of the 2017 Conference on Designing Interactive Systems. ACM, 587-598.

[13] Laura R Pina, Sang-Wha Sien, Teresa Ward, Jason C Yip, Sean A Munson, James Fogarty, and Julie A Kientz. [n. d.]. From Personal Informatics to Family Informatics: Understanding Family Practices around Health Monitoring. In CSCW. $2300-2315$. 